\title{
Caveolin-1 deficiency impairs synaptic transmission in hippocampal neurons
}

\author{
Soulmee Koh ${ }^{1}$, Wongyoung Lee ${ }^{1}$, Sang Myun Park ${ }^{2}$ and Sung Hyun Kim ${ }^{3,4^{*}}$ (D)
}

\begin{abstract}
In addition to providing structural support, caveolin-1 (Cav1), a component of lipid rafts, including caveolae, in the plasma membrane, is involved in various cellular mechanisms, including signal transduction. Although pre-synaptic membrane dynamics and trafficking are essential cellular processes during synaptic vesicle exocytosis/synaptic transmission and synaptic vesicle endocytosis/synaptic retrieval, little is known about the involvement of Cav1 in synaptic vesicle dynamics. Here we demonstrate that synaptic vesicle exocytosis is significantly impaired in Cav1-knockdown (Cav1-KD) neurons. Specifically, the size of the synaptic recycled vesicle pool is modestly decreased in Cav1-KD synapses and the kinetics of synaptic vesicle endocytosis are somewhat slowed. Notably, neurons rescued by triple mutants of Cav1 lacking palmitoylation sites mutants show impairments in both synaptic transmission and retrieval. Collectively, our findings implicate Cav1 in activity-driven synaptic vesicle dynamics — both exocytosis and endocytosis - and demonstrate that palmitoylation of Cav1 is important for this activity.
\end{abstract}

Keywords: Caveolin-1, Lipid raft, Synaptic vesicle exocytosis, Synaptic vesicle endocytosis, Synaptic transmission

\section{Introduction}

Lipid rafts are sphingolipid-cholesterol-enriched subcompartments in the plasma membrane with a size on the scale of several nanometers to micrometers [1]. Biochemically, they are detergent-insoluble complexes that also contain proteins, such as glycosylphosphatidylinositol (GPI)-anchored proteins and transmembrane proteins. Lipid rafts are known to be involved in a range of cellular functions, most notably including membrane trafficking and signaling. Lipid rafts also provide docking sites for membrane fusion [2]. Specifically, components of the membrane-fusion machinery (i.e., VAMP2, SNAP25, syntaxin1A) often localize to this membrane compartment and regulate exocytosis by interacting with lipid rafts [3].

A distinct type of lipid raft is caveolae, which are small (60-80 $\mathrm{nm}$ diameter) flask-shaped invaginations in

\footnotetext{
*Correspondence: sunghyunkim@khu.ac.kr

${ }^{3}$ Department of Physiology, School of Medicine, Kyung Hee University, Seoul 02447, South Korea

Full list of author information is available at the end of the article
}

membrane domains [4]. A major component of caveolae is caveolin (Cav), a family of a lipid raft-associated scaffolding proteins comprising Cav1, Cav2 and Cav3, which form homo- and heterodimers in lipid rafts [5]. Cav1 is a 178-amino-acid protein that binds to a fatty acid- and cholesterol-containing microdomain via palmitoyl tails on three cysteine residues, C133, C143, and C156. Cav1 is a major constituent of caveolae, which are present in various cell types, although they are generally less abundant in neurons [6]. Notably, some reports have suggested that Cav1 plays a role in neurons distinct from its function in caveolae [7].

The presynaptic membrane is a highly dynamic area that is important for the proper function of neurons. For example, activity-driven synaptic vesicle fusion and recycling in nerve terminals is an essential process underlying synaptic communication. When an action potential arrives at a nerve terminal, synaptic vesicle membranes fuse to the plasma membrane. Thereafter, the plasma membrane invaginates, reforming synaptic vesicles via an endocytic process. Given the prominence of Cav1 in plasma membrane domains and the essential role of

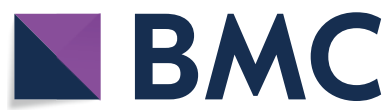

(c) The Author(s) 2021. Open Access This article is licensed under a Creative Commons Attribution 4.0 International License, which permits use, sharing, adaptation, distribution and reproduction in any medium or format, as long as you give appropriate credit to the original author(s) and the source, provide a link to the Creative Commons licence, and indicate if changes were made. The images or other third party material in this article are included in the article's Creative Commons licence, unless indicated otherwise in a credit line to the material. If material is not included in the article's Creative Commons licence and your intended use is not permitted by statutory regulation or exceeds the permitted use, you will need to obtain permission directly from the copyright holder. To view a copy of this licence, visit http://creativecommons.org/licenses/by/4.0/. The Creative Commons Public Domain Dedication waiver (http://creativeco mmons.org/publicdomain/zero/1.0/) applies to the data made available in this article, unless otherwise stated in a credit line to the data. 
membrane dynamics in nerve terminals, it is reasonable to infer that Cav1 might be involved in this dynamic process.

In this study, we monitored synaptic vesicle dynamics in the absence of Cav1. Neurons lacking Cav1 exhibited impaired synaptic vesicle exocytosis, manifesting as suppressed synaptic transmission and a decreased rate of synaptic vesicle exocytosis. We further found that synaptic vesicle endocytosis is also altered in Cav1-depleted neurons. Finally, we demonstrated that palmitoylation of Cav1 is important for proper synaptic function.

\section{Methods}

\section{Primary neuron culture}

Hippocampal CA1-CA3 regions were isolated from neonatal (0-1 day old) Sprague-Dawley rats (DBL, Strain code: NTac:SD) and plated on poly-ornithinecoated coverslips. Neurons were transfected 8 days after plating and further incubated for 14-21 days in culture medium, as described previously [8]. Neurons were imaged 14-21 days after plating. All results are from at least three independent primary cultures. All animal procedures in this study were performed in accordance with the Guide for the Care and Use of Laboratory Animals with the approval of the Institutional Animal Care and Use Committee of Kyung Hee University.

\section{Plasmids and transfection}

Small hairpin (inhibitory) RNA against Cav1 (shRNACav1) with the targeting sequence, $5^{\prime}$-GAT TGA TCT GGT CAA CCG C-3' [9], was synthesized, annealed, and ligated into the pSuper vector according to the manufacturer's instructions. The palmitoylation sites-deleted Cav1 triple mutant, Cav1-C133A/C143A/C156A [10], was constructed by site-directed mutagenesis kit (Stratagene) using mCherry-tagged WT Cav1 (Cav1-mCh) as a template. For optical imaging, primary cultured hippocampal neurons were transfected with the indicated constructs using the $\mathrm{Ca}^{2+}$ phosphate precipitation method, as previously described [8]. Briefly, plasmids were incubated with 2 X HEBS $(273 \mathrm{mM} \mathrm{NaCl}, 9.5 \mathrm{mM}$ $\mathrm{KCl}, 1.4 \mathrm{mM} \mathrm{Na}_{2} \mathrm{HPO}_{4} \cdot 7 \mathrm{H}_{2} \mathrm{O}, 15 \mathrm{mM}$ D-glucose, $42 \mathrm{mM}$ HEPES pH 7.10) containing $2 \mathrm{mM} \mathrm{Ca}^{2+}$, after which the mixture was applied to hippocampal neurons cultured for 8 days in vitro (DIV8). For western blot analysis, primary neurons were transfected by electroporation using a NEPA21 system, as described by the manufacturer (Bulldog Bio).

\section{Immunofluorescence}

For immunofluorescence analyses, DIV14-18 neurons were fixed with $4 \%$ paraformaldehyde, permeabilized with $0.2 \%$ Triton $\mathrm{X}-100$, and blocked with $5 \%$ bovine serum albumen (BSA). After incubating with anti-synapsin1 primary antibodies (BD Bioscience), neurons were incubated with Alexa 488-conjugated secondary antibodies (Invitrogen).

\section{Lipid raft labeling}

Neuronal lipid rafts were labeled by incubating DIV14-18 neurons with the lipid raft marker, GM1, using a Vybrant Alexa Fluor 594 Lipid Raft Labeling Kit (Thermo Fisher) according to the manufacturer's instructions. Briefly, neurons were incubated for $30 \mathrm{~min}$ at $4{ }^{\circ} \mathrm{C}$ with Alexa594-conjugated cholera toxin subunit B (CT-B), which specifically binds to the plasma membrane ganglioside, GM1, an integral part of lipid rafts. Thereafter, cells were incubated with an anti-CT-B antibody for $30 \mathrm{~min}$ at $4{ }^{\circ} \mathrm{C}$ [11].

\section{Optical imaging}

For immunofluorescence, images of fixed neurons were acquired using a Leica DMRBE microscope with a PL Fluor 40x (1.0 NA.) objective equipped with a CoolSNAP HQ camera (Photometric) driven by MetaMorph software. For pHluorin assays in nerve terminals, live-cell imaging was performed on DIV14-21 neurons transfected with Physin-pH and the indicated plasmids 8 days after plating. Coverslips were mounted in a laminar-flow-perfused stimulation chamber on the stage of a custom-built, laser-illuminated epifluorescence microscope (Zeiss Observer). Live-cell images were acquired with an Andor iXon Ultra 897 (Model \#DU-897U-CS0-\#BV) back-illuminated EMCCD camera. A diode-pumped OBIS 488 laser (Coherent), shuttered by synchronizing the TTL on/off signal from the EMCCD camera during acquisition, was utilized as a light source. Fluorescence excitation/emission and collection were achieved using a $40 \times$ Fluar Zeiss objective lens (1.3 NA) and 500-550 nm emission and $498 \mathrm{~nm}$ dichroic filters (Chroma). Action potentials (APs) were evoked by passing a 1 -ms current pulse through platinumiridium electrodes from an isolated current stimulator (World Precision Instruments). Neurons were perfused with Tyrode's buffer consisting of $119 \mathrm{mM}$

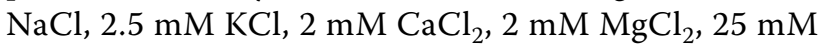
HEPES, $30 \mathrm{mM}$ glucose, $10 \mu \mathrm{M}$ 6-cyano-7-nitroquinoxaline-2,3-dione (CNQX), and $50 \mu \mathrm{M} \mathrm{D}, \mathrm{L}-2$-amino5-phosphonovaleric acid (AP5), adjusted to $\mathrm{pH}$ 7.4. All experiments were carried out at $30{ }^{\circ} \mathrm{C}$; all images were acquired at $2 \mathrm{~Hz}$ with a $50-\mathrm{ms}$ exposure; and all chemicals were purchased from Sigma unless otherwise specified. 


\section{Image analysis}

The results of pHluorin-based assays were analyzed as previously described [12], with minor modifications. Images were analyzed using Image J (http://rsb.info. nih.gov/ij) with the Time Series Analyzer plugin, available at https://imagej.nih.gov/ij/plugins/time-serie s.html. Differences in the expression levels of Cav1 in the presence and absence of shRNA-Cav1 were measured using Image J. Synaptic boutons of neurons were selected as oval regions of interest (diameter, 10 pixels), and the intensity of pHluorin-based fluorescence at synapses was measured and analyzed using Origin Pro 2020. The kinetics of endocytosis and exocytosis were fitted using a single exponential decay function.

\section{Western blotting}

Hippocampal CA3-CA1 regions were dissected from 1-day-old Sprague-Dawley rats, dissociated, plated onto poly-ornithine-coated 6-well dishes, and incubated for 14-18 days. At DIV8, neurons in three wells were transfected with control plasmids and neurons in the other three wells were transfected with shRNACav1. Cells were further incubated until DIV14-18. All experiments were performed in parallel under the same conditions. Cells were lysed with lysis buffer containing $10 \mathrm{mM}$ Tris (pH 7.4), 1\% SDS, $10 \mathrm{mM} \mathrm{NaF}$ and $1 \mathrm{mM}$ PMSF, supplemented with a protease inhibitor mixture (Complete Mini; Roche, Germany). The protein concentration in lysates was determined using a bicinchoninic acid (BCA) assay (Thermo, IL). Lysate samples containing equal amounts of protein were subjected to sodium dodecyl sulfate-polyacrylamide gel electrophoresis (SDS-PAGE) and subsequently transferred to polyvinylidene difluoride (PVDF) membranes. Membranes were blocked by incubating with $5 \%$ nonfat dry milk, and subsequently incubated with anti-Cav1 (BD Biosciences) and anti- $\beta$-actin (Santa Cruz, CA) primary antibodies. Band intensities were quantified densitometrically, and the $[\mathrm{Cav} 1] /[\beta$-actin $]$ ratio was measured after background subtraction.

\section{Statistical analyses}

Statistical analyses were performed using either oneway analysis of variance (ANOVA) or Student's t test, as appropriate. Error bars indicate standard errors of the mean (SEM).

\section{Results}

\section{Cav1 is localized to nerve terminals in primary cultured hippocampal neurons}

The function of Cav1 as a component of lipid rafts has not been extensively explored in the nervous system, reflecting the fact that its existence and localization in the membrane of neurons has not been firmly established [6]. Before investigating the physiological role of Cav1 in synapses, we assessed the lipid raft localization of Cav1. First, Cav1-mCh together with the presynaptic marker, synaptophysin-GFP, was introduced into primary cultured hippocampal neurons. After further incubating and then fixing neurons at 14-18 days in vitro (DIV1418), synaptic co-localization of Cav1 with the presynaptic marker, synaptophysin, was assessed. This assessment showed that Cav1 is highly co-localized with synaptophysin (Fig. 1a), a finding confirmed by correlation analyses (Fig. 1b). We next assessed the distribution of lipid rafts at synapses by incubating neurons with cholera toxin $\mathrm{B}(\mathrm{CTxB})$, a known lipid raft-specific marker [13], and labeling synapses using an anti-synapsin I antibody. The distribution of $\mathrm{CTxB}$ closely corresponded with that of anti-synapsin 1 signals (Fig. 1c, d), suggesting that Cav1 is indeed localized to synapses.

\section{Cav1 is involved in synaptic vesicle exocytosis at CNS synapses}

To investigate the functional role of Cav1 at synapses, we first constructed shRNA targeting Cav1 (shRNA-Cav1) for Cav1 knockdown (KD) in cultured neurons. shRNA-Cav1 was delivered into neurons by electroporation, and the efficacy of Cav1 knockdown was assessed by western blotting (Fig. 2a), which showed that Cav1 expression in Cav1-KD neurons was decreased to $\sim 20 \%$ of that in parental neurons (Fig. 2b). Next, we monitored synapse function using the Synaptophysin-pHluorin (Physin-pH) system, which has been used effectively to monitor the presynaptic physiological processes of synaptic vesicle exocytosis (synaptic transmission) and synaptic vesicle endocytosis (synaptic retrieval) [14]. First, to verify that Cav1 is involved in synaptic transmission, we cotransfected neurons with Physin-pH and shRNA-Cav1 at DIV8 and 1 week thereafter monitored synaptic transmission in response to stimulation of neurons with 100 action potentials (APs) at $10 \mathrm{~Hz}$. Interestingly, activity-driven synaptic vesicle exocytosis was strongly suppressed in Cav1-KD neurons, which exhibited responses that were $\sim 50 \%$ of those in controls (Fig. 2c-e). Consistent with this, single synaptic bouton analyses revealed a significant decrease in the distribution of individual bouton responses in Cav1KD neurons (Fig. 2f, g), implicating Cav1 in the synaptic transmission process.

To determine whether the synaptic transmission defect in Cav1-KD neuron is a direct result of the Cav1 deficiency, we performed rescue experiments in which we assessed synaptic vesicle exocytosis in response to stimulation with $100 \mathrm{APs}$ at $10 \mathrm{~Hz}$ in Cav1-KD 
a

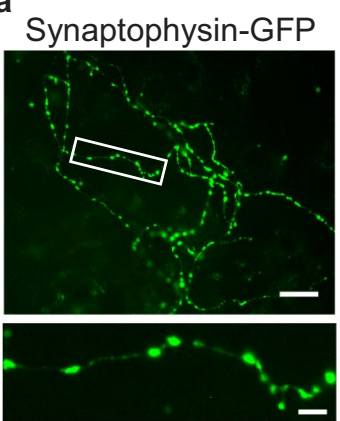

C

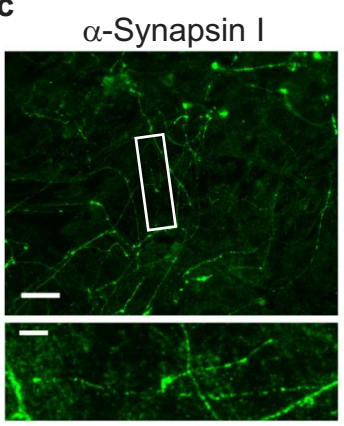

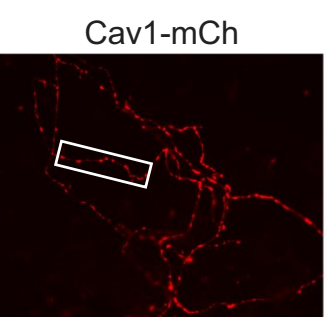

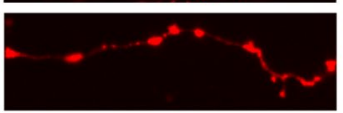

$\alpha$-Cholera toxin B
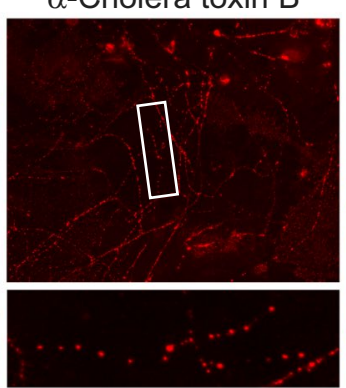

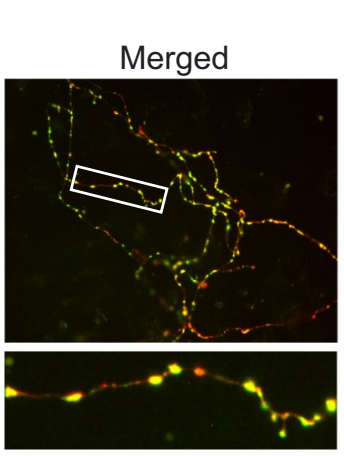

b

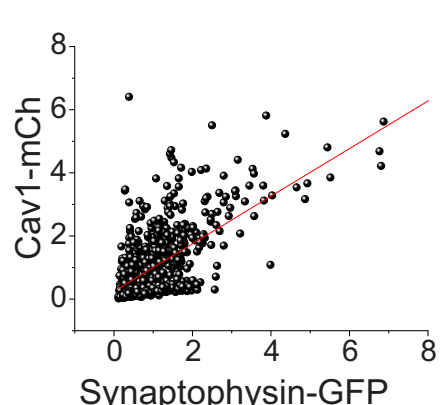

d
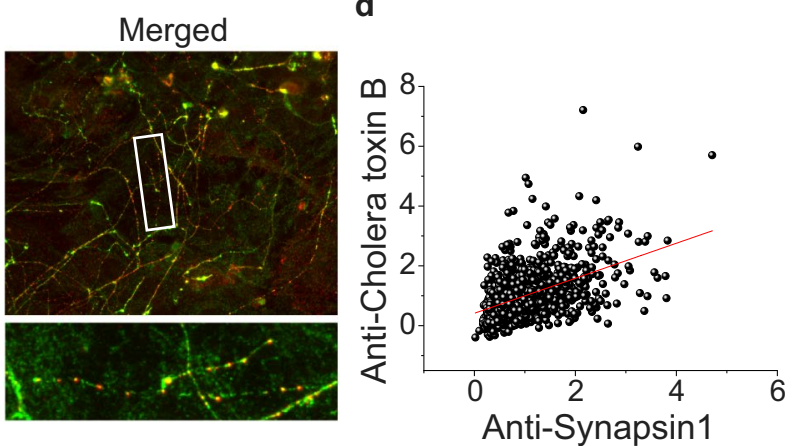

Fig. 1 Cav1 co-localizes with lipid rafts at nerve terminals in primary cultured hippocampal neurons. a Representative images of exogenous synaptophysin-GFP (left), a nerve terminal marker, together with exogenous Cav1-mCherry (Cav1-mCh; middle) in primary cultured hippocampal neurons. Neurons were co-transfected with synaptophysin-GFP and Cav1-mCh at DIV8 and fixed at DIV14-18. b Correlation between synaptophysin-GFP and Cav1-mCh expression levels. c Representative images of synapsin I and lipid rafts (CT-B) in primary cultured hippocampal neurons. Neurons were stained with Alexa-Fluor 594-conjugated CT-B (red) and its antibodies, and subsequently immunostained using an anti-synapsin I (green) antibody (SYSY) and Alexa-labeled secondary antibody (Alexa-488). $\mathbf{d}$ Correlation between synapsin I and CT-B expression levels. Scale bar: $10 \mu \mathrm{m}$ (up) and $2 \mu \mathrm{m}$ (bottom)

neurons exogenously expressing shRNA-insensitive Cav1. These experiments clearly showed that exogenous expression of shRNA-insensitive Cav1 restored synaptic transmission to approximately control levels in neurons lacking endogenous Cav1 (Fig. 2c-g and Additional file 1: Fig. S1), demonstrating that Cav1 is indeed involved in synaptic vesicle exocytosis in hippocampal neurons.

\section{Synaptic vesicle exocytosis rate is decreased in Cav1-KD neurons}

Because synaptic vesicle release is suppressed in Cav1KD neurons, we next tested whether the kinetics of synaptic vesicle exocytosis, which is a continuous synaptic transmission process, is impacted in Cav1-KD neurons. Neurons transfected with Physin-pH with or without shRNA-Cav1 were subjected to prolonged stimulation in the presence of the endocytosis inhibitor bafilomycin, which allowed us to monitor the exocytosis process in isolation [15]. As expected, synaptic vesicle exocytosis was significantly slowed ( 1.8 fold) in Cav1-KD neurons compared with control neurons. In addition, the functional synaptic pool of recycling vesicles was also slightly decreased in Cav1-KD neurons. These results suggest that Cav1 is also involved in the synaptic vesicle release process and synaptic vesicle pool function.

\section{Synaptic vesicle endocytosis rate is slowed in Cav1-KD neurons}

Next, we determined whether Cav1 is also involved in the synaptic vesicle endocytosis process. To demonstrate the role of Cav1 in synaptic retrieval, we again applied a pHluorin-based assay. In these experiments, Cav1-KD neurons co-expressing Physin-pH were stimulated with 100 or 300 APs at $10 \mathrm{~Hz}$ and then the synaptic vesicle endocytic process was assessed by monitoring the decay of Physin-pH fluorescence and fitting the results to a single exponential decay function. This analysis showed that a Cav1 deficiency in 
(See figure on next page.)

Fig. 2 Synaptic vesicle exocytosis is slowed in Cav1-KD neurons. a Representative images of western blotting. Neurons transfected with shRNA-Cav1 were lysed, after which proteins in lysates were resolved by SDS-PAGE and transferred to PVDF membranes. Membranes were subsequently incubated with anti-Cav1 antibody and anti- $\beta$-actin antibody. b Cav1 expression levels in control and Cav1-KD neurons. Intensities of Cav1 bands expressed relative to those of $\beta$-actin are presented as means $\pm S E M s$. ([Cav1 $\left.{ }_{\text {Con }}\right]=100.00 \pm 6.8 ;\left[\operatorname{Cav}_{\mathrm{KD}}\right]=22.45 \pm 3.2, \mathrm{n}=4$ ). c Representative images of Physin-pH at synaptic boutons in Control, Cav1-KD, and Cav1-Rescue (Cav1-Res) neurons under basal conditions (top), with stimulation at $10 \mathrm{~Hz}$ for $10 \mathrm{~s}$ (100 APs; middle), and following application of $\mathrm{NH}_{4} \mathrm{Cl}$ (bottom). Scale bar: $10 \mu \mathrm{m}$. $\mathbf{d}$ Representative trace of Physin-pH responses to 100 APs in Control (black), Cav1-KD (red), and Cav1-Res (green) neurons. e Quantification of synaptic transmission, expressed as means \pm SEMs in Control $(0.15 \pm 0.02 ; n=14$ cells), Cav1-KD $(0.07 \pm 0.01 ; n=14$ cells) and Cav1-Res $(0.14 \pm 0.02 ; n=7$ cells $)$ neurons $\left({ }^{* * *} p<0.001,{ }^{* *} p<0.01\right)$. f and $\mathbf{g}$ Cumulative frequency of exocytosis from single synaptic bouton analyses of Control, Cav1-KD, and Cav1-Res neurons

neurons slowed the kinetics of synaptic vesicle endocytosis following AP stimuli (100 or 300 APs) (Fig. 3), decreasing the endocytosis rate by $\sim 1.5$-fold compared with control neurons (Fig. 4).

\section{Palmitoylation of Cav1 is involved in Cav1 modulation of synaptic function}

Palmitoylation-the covalent attachment of palmitic acid to cysteine-is among the protein modifications that mediate membrane association [16]. Cav1 has three cysteine sites, C133, C143 and C156, for palmitoylation, which is critical for attachment of Cav1 to the membrane. With this in mind, we examined whether palmitoylation of Cav1 is necessary for Cav1 modulation of synaptic function. To this end, we mutated the three palmitoylation sites, C133, C143 and C156, in Cav1 to alanine, yielding the non-palmitoylatable triple-mutant (tri-mut), Cav1-C133A/C143A/C156A. To determine whether palmitoylation of Cav1 influences functional synaptic physiology, we replaced endogenous Cav1 with the Cav1 tri-mut by co-transfecting neurons with shRNA-Cav1, Cav1 tri-mut and PhysinpH (Fig. 5i, j and Additional file 1: Fig. S1). We then monitored synaptic vesicle exocytosis and endocytosis in response to stimulation with $100 \mathrm{APs}$ at $10 \mathrm{~Hz}$. As shown in Fig. 5a, b, synaptic vesicle exocytosis in Cav1 tri-mut-expressing neurons was decreased to $\sim 6 \%$ of that in control neurons expressing endogenous WT Cav1 only. Furthermore, the rate of synaptic vesicle fusion was significantly increased ( twofold) compared with control neurons (Fig. 5e, f), although the recycling pool size of synaptic vesicles was not changed (Fig. $5 \mathrm{~g}, \mathrm{~h}$ ), suggesting that palmitoylation-dependent membrane localization of Cav1 is important for synaptic transmission. In addition, the rate of synaptic vesicle endocytosis in Cav1 tri-mut-expressing neurons was also increased $\sim 1.5$ fold compared with the endocytosis rate in controls (Fig. 5c, d). Collectively, these results indicate that palmitoylation of Cav1 has a critical role in synaptic physiology, impacting both synaptic vesicle exocytosis and endocytosis. Interestingly, despite the absence of palmitoylation, the Cav1 tri-mut was still distributed to the presynaptic area (Fig. 5i, j).

\section{Discussion}

Lipid rafts are specific compartments of the plasma membrane that are known to be involved in membrane trafficking and cellular singling [1]. Cav1 is a key protein in lipid rafts, including caveolae, acting as a scaffolding protein to provide structural support. Cav1 is expressed in various cell types, where its involvement in a number of cell processes, including cellular trafficking and signaling, have been studied [17]; however, Cav1 has been less thoroughly investigated in the central nervous system (CNS).

In the present study, we demonstrated that Cav1 is involved in presynaptic processes. We found that synaptic transmission was significantly suppressed in Cav1-KD neurons, generated by introducing shRNA targeting Cav1, as evidenced by approximately a $50 \%$ decrease in synaptic vesicle exocytosis compared with control neurons. This defect in synaptic vesicle 
a

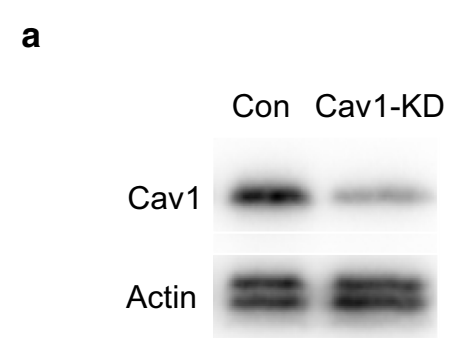

Cav1

Actin

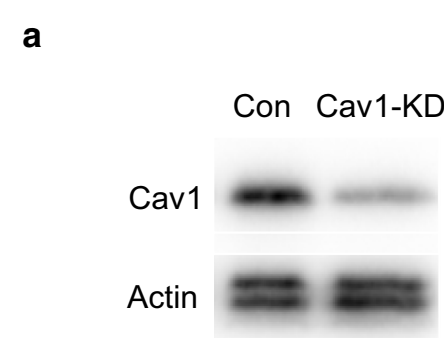

Actin

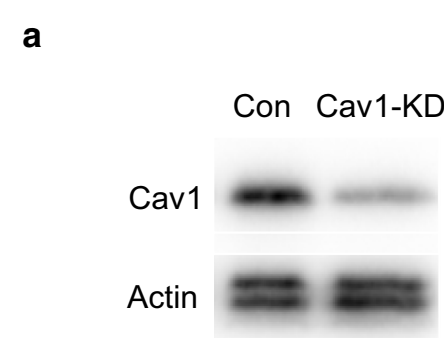$$
\text { . }
$$

\section{b}

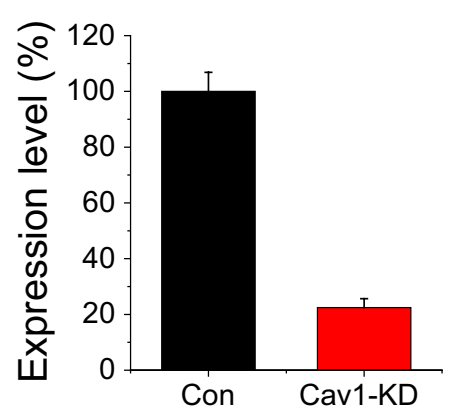

c

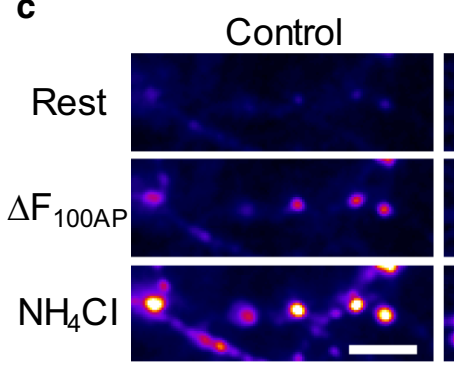

Cav1-KD

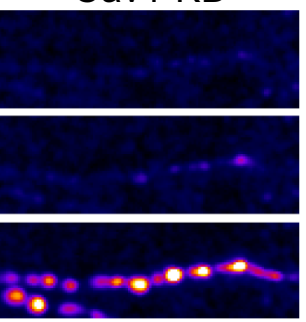

Cav1-Res
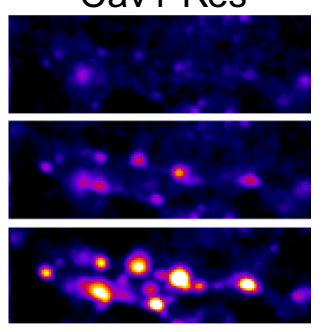

d

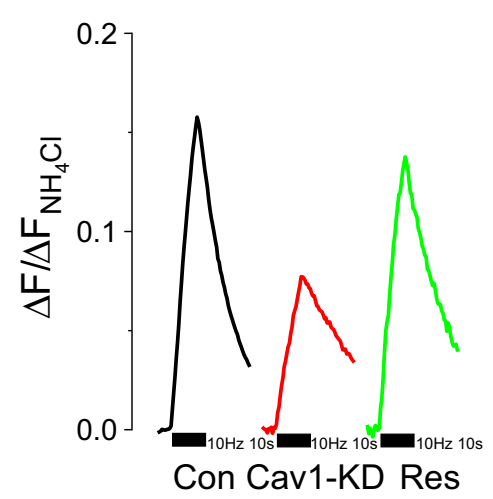

e

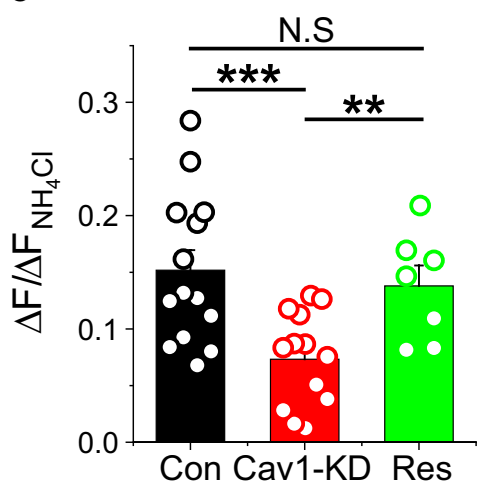

f

9
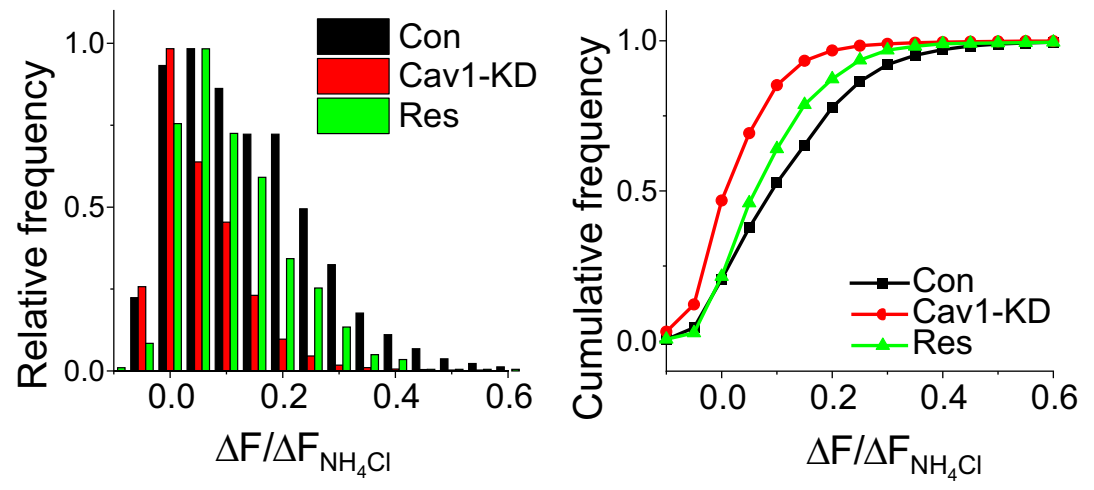

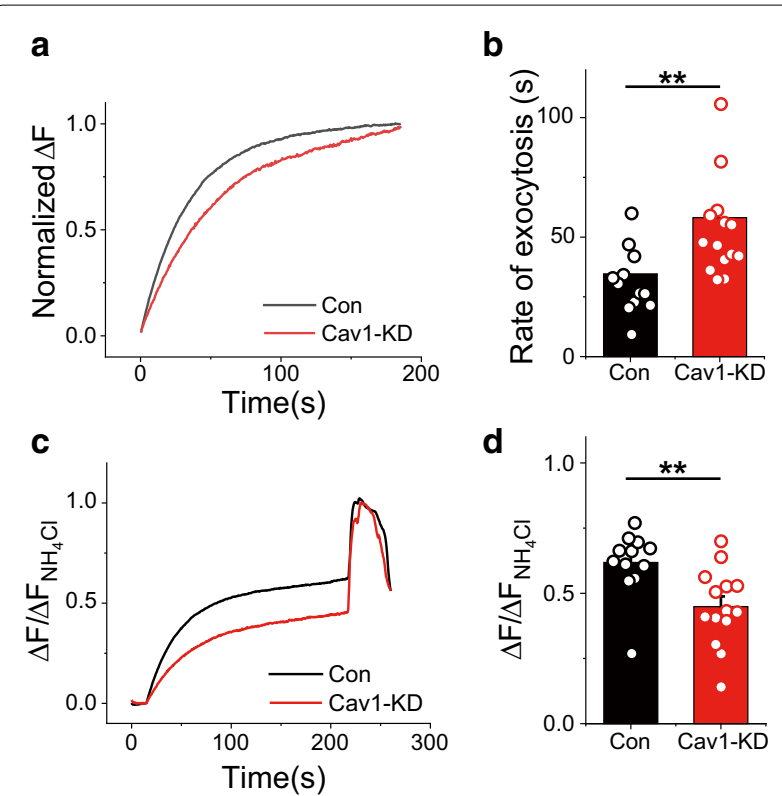

Fig. 3 Synaptic vesicle exocytosis rate is significantly decreased in Cav1-KD neurons. a Representative normalized traces of Physin-pH exocytosis from Control (black) and Cav1-KD (red) neurons. b Quantification of exocytosis, expressed as mean values of time constants $(\tau) \pm S E M$, in Control $\left(\tau_{\text {exo }}, 34.61 \pm 4.23 ; n=12\right.$ cells) and Cav1-KD ( $\tau_{\text {exo, }}, 58.12 \pm 5.84 ; \mathrm{n}=14$ cells) neurons. $\mathbf{c}$ Representative traces of Physin-pH responses to 2000 APs at $10 \mathrm{~Hz}$ in the presence of bafilomycin A1 (BAF) in control and Cav1-KD neurons. d Quantification of recycling vesicle pool size expressed as means \pm SEMs in Control $(0.62 \pm 0.04 ; n=12$ cells $)$ and Cav1-KD $(0.45 \pm 0.04 ; n=14$ cells) neurons (** $p<0.01)$

exocytosis was rescued by re-introducing an shRNAinsensitive form of Cav1 into Cav1-KD neurons. In addition, the speed of synaptic vesicle fusion was also impaired in Cav1-KD neurons. We further assessed the synaptic vesicle endocytosis process, showing that the kinetics of synaptic vesicle endocytosis was modestly slowed in Cav1-KD neurons compared with control neurons. Similar alterations of synaptic vesicle trafficking were observed in Cav1-KD cells expressing the
Cav1 tri-mut, lacking three palmitoylatable cysteines, indicating that palmitoylation is essential for normal Cav1 function. Collectively, these findings implicate palmitoylated Cav1 in synaptic vesicle trafficking at CNS synapses (Additional file 2: Fig. S2 and Additional file 3: Fig. S3).

Although this study has uncovered some important functions of Cav1 in presynaptic terminals, several questions remain to be addressed. First, how does Cav1 affect synaptic transmission? The expectation is that this may depend on the membrane composition of the release site. It has been reported that lipid rafts regulate exocytosis through spatial regulation of SNARE complexes, which are responsible for vesicle fusion [1]. This would suggest that Cav1 might control lipid raft formation and distribution of SNARE complexes in lipid rafts. Consistent with this, Cav1 function can also be linked to the synaptic vesicle endocytosis process. It is also possible that Cav1 controls the lipid content of synaptic vesicles, a supposition that would require a careful analysis of the lipid components of synaptic vesicles. Alternatively, Cav1 may associate with synaptic vesicle proteins. The next question arising is how does Cav1 dynamically regulate lipid rafts at synapses and is this regulation related to neural activity? In this context, Cav1-dependent lipid rafts of various sizes are incorporated into synaptic vesicles during endocytosis, and Cav1 is able to interact with endocytic components, which modulates synaptic vesicle endocytosis. A further question is whether the synaptic function of Cav1 related to a neural disorder. Studies have revealed that Cav1 or lipid rafts are implicated in neurodegenerative diseases such as Alzheimer's disease (AD) and Parkinson's disease (PD) [18-20], and that Cav1 is also involved in non-neural diseases such as cancer and diabetes $[21,22]$. The remaining questions are challenging, but emerging high-resolution imaging (e.g. super-resolution microscopy) technology might provide an approach for addressing them. 

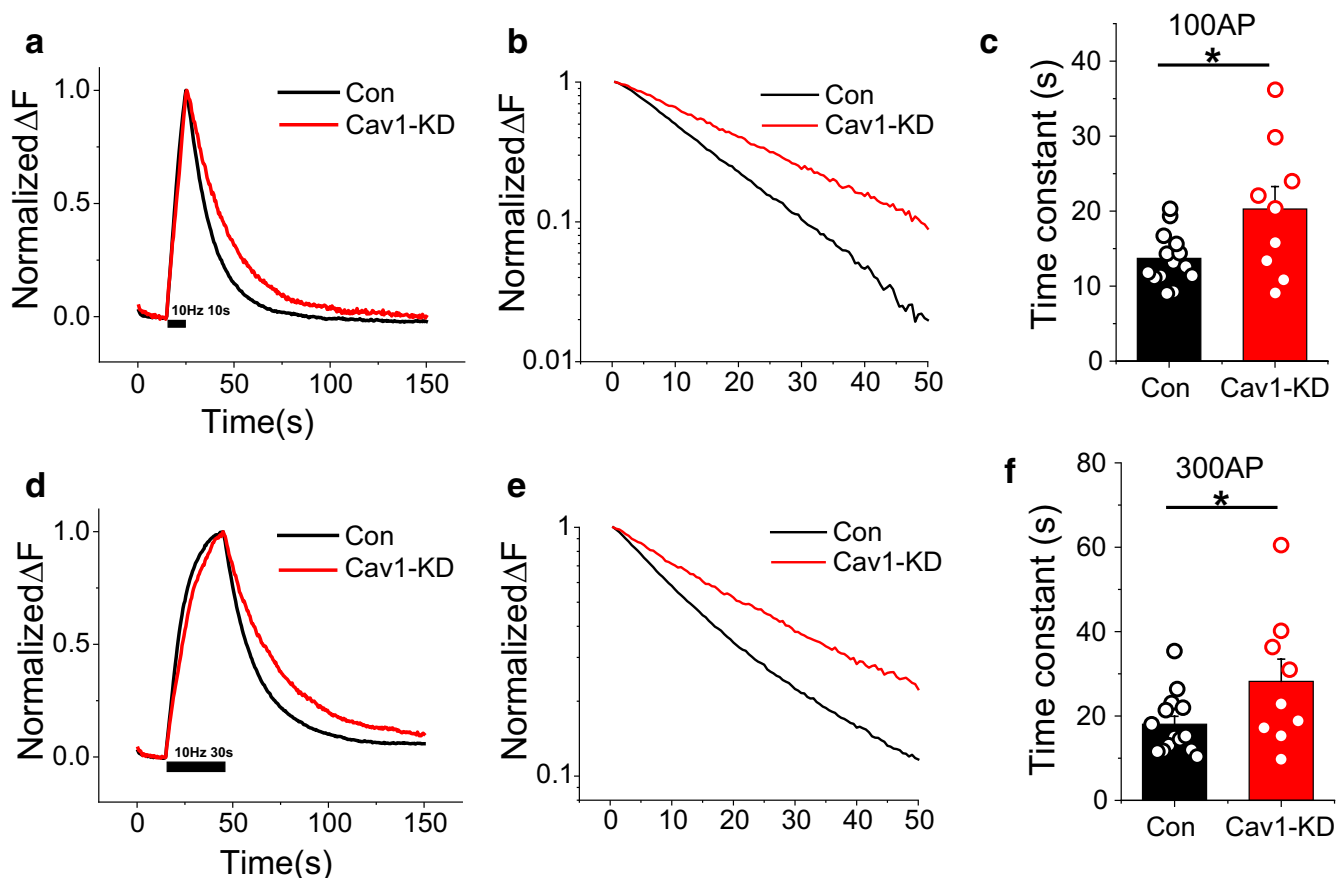

Fig. 4 Synaptic vesicle endocytosis kinetics are slowed in Cav1-KD neurons. a, d Representative traces of Physin-pH responses to 100 APs (a) and 300 APs (d) in Control and Cav1-KD neurons. Neurons transfected with Physin-pH with or without shRNA-Cav1 were stimulated with 100 or 300 APs at $10 \mathrm{~Hz}$. b, e The traces of Physin-pH responses to 100 APs (b) and 300 APs (e) were projected to the semi-log scale for clearance. (C, D) Quantification of post-stimulus endocytic time constants, expressed as means \pm SEMs in (c) Control ( $\tau_{\text {endo, }} 13.71 \pm 0.92 ; n=14$ cells $)$ and Cav1-KD ( $\tau_{\text {endo, }} 20.29 \pm 2.99 ; n=9$ cells) neurons stimulated with 100 APs, and (d) Control ( $\tau_{\text {endor }}$ 18.04 $\pm 1.90 ; n=14$ cells) and Cav1-KD ( $\tau_{\text {endor }}$ 28.21 \pm 5.28 ; $n=9$ cells) neurons stimulated with $300 \operatorname{APs}\left({ }^{*} p<0.05\right)$ 


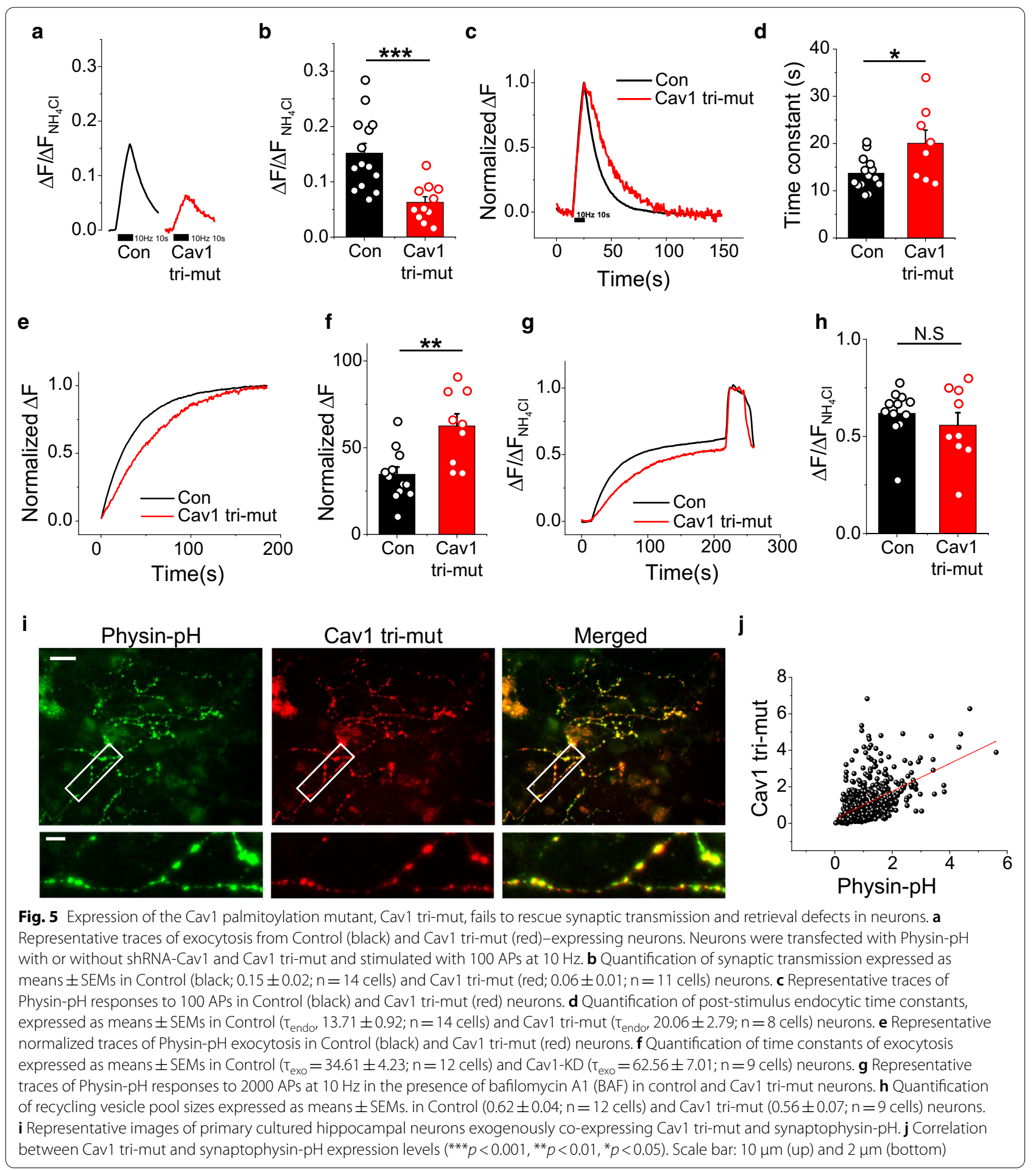




\section{Abbreviations}

CT-B: Cholera toxin subunit B; Physin-pH: Synaptophysin-pHluorin; Cav1: Caveolin-1; BAF: Bafiliomycin1; KD: Knock-down

\section{Supplementary Information}

The online version contains supplementary material available at https://doi. org/10.1186/s13041-021-00764-z.

Additional file 1: Fig S1. shRNA-insensitive Cav1 was successfully expressed in Cav1-KD neurons.

Additional file 2: Fig S2. Raw data for western blot.

Additional file 3: Fig S3. Raw data for other numeric data.

\section{Acknowledgements}

We would like to thank Prof. Sang Myun Park for the generous gift of Cav1$\mathrm{mCh}$ plasmid. We also thank members of the Kim laboratory for their valuable comments and discussion.

\section{Authors' contributions}

SK, WL, and SHK performed the experiments and data analysis. SMP and SHK designed the experiment and analyzed data. SK and SHK wrote the manuscript. SHK conducted all of the study. All authors read and approved the final manuscript.

\section{Funding}

This work was supported by the NSF (National Science Foundation) of Korea (NRF-2016M3C7A1905072, 2017M3C7A1048268, 2018R1A6A03025124, 2020R1A2C2010791).

\section{Availability of data and materials}

Please contact author for data requests.

\section{Declarations}

\section{Ethics approval and consent to participate}

All methods were carried out in accordance with relevant guidelines and regulations, and the study was approved by the animal ethics committee of Kyung Hee University.

\section{Consent for publication}

Not applicable.

\section{Competing interests}

The authors declare that they have no competing interests.

\section{Author details}

${ }^{1}$ Department of Neuroscience, Graduate School, Kyung Hee University, Seoul 02447, South Korea. ${ }^{2}$ Department of Pharmacology, Ajou University School of Medicine, Suwon 16499, South Korea. ${ }^{3}$ Department of Physiology, School of Medicine, Kyung Hee University, Seoul 02447, South Korea. ${ }^{4}$ Medical Research Center for Bioreaction To Reactive Oxygen Species and Biomedical Science Institute, School of Medicine, Kyung Hee University, Seoul 02447, South Korea.

Received: 10 December 2020 Accepted: 25 February 2021

Published online: 16 March 2021

\section{References}

1. Simons K, Ikonen E. Functional rafts in cell membranes. Nature. 1997:387(6633):569-72.

2. Chamberlain LH, Burgoyne RD, Gould GW. SNARE proteins are highly enriched in lipid rafts in PC12 cells: implications for the spatial control of exocytosis. Proc Natl Acad Sci USA. 2001;98(10):5619-24.

3. Salaun C, James DJ, Chamberlain LH. Lipid rafts and the regulation of exocytosis. Traffic. 2004;5(4):255-64.
4. Smart EJ, Graf GA, MCNiven MA, Sessa WC, Engelman JA, Scherer PE, et al. Caveolins, liquid-ordered domains, and signal transduction. Mol Cell Biol. 1999;19(11):7289-304.

5. Campos A, Burgos-Ravanal R, Gonzalez MF, Huilcaman R, Lobos Gonzalez L, Quest AFG. Cell intrinsic and extrinsic mechanisms of caveolin-1-enhanced metastasis. Biomolecules. 2019;9(8):314.

6. Shikanai M, Nishimura YV, Sakurai M, Nabeshima YI, Yuzaki M, Kawauchi T. Caveolin-1 promotes early neuronal maturation via caveolae-independent trafficking of $\mathrm{N}$-cadherin and L1. iScience. 2018;7:53-67.

7. Head BP, Insel PA. Do caveolins regulate cells by actions outside of caveolae? Trends Cell Biol. 2007;17(2):51-7.

8. Kyung JW, Bae JR, Kim DH, Song WK, Kim SH. Epsin1 modulates synaptic vesicle retrieval capacity at CNS synapses. Sci Rep. 2016;6:31997.

9. Ge S, Pachter JS. Caveolin-1 knockdown by small interfering RNA suppresses responses to the chemokine monocyte chemoattractant protein-1 by human astrocytes. J Biol Chem. 2004;279(8):6688-95.

10. Uittenbogaard A, Smart EJ. Palmitoylation of caveolin-1 is required for cholesterol binding, chaperone complex formation, and rapid transport of cholesterol to caveolae. J Biol Chem. 2000;275(33):25595-9.

11. Raghu H, Sharma-Walia N, Veettil MV, Sadagopan S, Caballero A, Sivakumar R, et al. Lipid rafts of primary endothelial cells are essential for Kaposi's sarcoma-associated herpesvirus/human herpesvirus 8-induced phosphatidylinositol 3-kinase and RhoA-GTPases critical for microtubule dynamics and nuclear delivery of viral DNA but dispensable for binding and entry. J Virol. 2007;81(15):7941-59.

12. Kim SH, Ryan TA. Balance of calcineurin Aalpha and CDK5 activities sets release probability at nerve terminals. J Neurosci . 2013;33(21):8937-50.

13. Day CA, Kenworthy AK. Functions of cholera toxin B-subunit as a raft cross-linker. Essays Biochem. 2015:57:135-45.

14. Bae JR, Lee W, Jo YO, Han S, Koh S, Song WK, et al. Distinct synaptic vesicle recycling in inhibitory nerve terminals is coordinated by SV2A. Prog Neurobiol. 2020;194:101879.

15. Kyung JW, Kim JM, Lee W, Ha TY, Cha SH, Chung KH, et al. DJ-1 deficiency impairs synaptic vesicle endocytosis and reavailability at nerve terminals. Proc Natl Acad Sci USA. 2018;115(7):1629-34

16. Linder ME, Deschenes RJ. Palmitoylation: policing protein stability and traffic. Nat Rev Mol Cell Biol. 2007:8(1):74-84

17. Parton RG, Simons K. The multiple faces of caveolae. Nat Rev Mol Cell Biol. 2007;8(3):185-94

18. Gaudreault SB, Dea D, Poirier J. Increased caveolin-1 expression in Alzheimer's disease brain. Neurobiol Aging. 2004;25(6):753-9.

19. Cha SH, Choi YR, Heo CH, Kang SJ, Joe EH, Jou I, et al. Loss of parkin promotes lipid rafts-dependent endocytosis through accumulating caveolin-1: implications for Parkinson's disease. Mol Neurodegener. 2015;10:63.

20. Head BP, Peart JN, Panneerselvam M, Yokoyama T, Pearn ML, Niesman IR, et al. Loss of caveolin-1 accelerates neurodegeneration and aging. PLoS ONE. 2010;5(12):e15697.

21. Nwosu ZC, Ebert MP, Dooley S, Meyer C. Caveolin-1 in the regulation of cell metabolism: a cancer perspective. Mol Cancer. 2016;15(1):71.

22. Haddad D, Al Madhoun A, Nizam R, Al-Mulla F. Role of caveolin-1 in diabetes and its complications. Oxid Med Cell Longev. 2020;2020:9761539.

\section{Publisher's Note}

Springer Nature remains neutral with regard to jurisdictional claims in published maps and institutional affiliations. 